\title{
DERECHO HUMANO AL TRABAJO. ESTUDIO DE CASO: LOS DERECHOS DE LOS TRABAJADORES DEL SECTOR SALUD EN EL MUNICIPIO DE SOLEDAD-ATLÁNTICO, ColOMBIA
}

\author{
Sandra Irina Villa Villa \\ Juan Carlos Berrocal Duran ${ }^{2}$
}

\section{Resumen}

Los conceptos de Trabajo decente y Trabajo digno, son utilizados como sinónimos, sin embargo, tienen fuentes normativas diferentes. El enfoque de Trabajo Decente ha sido desarrollado ampliamente por la Organización Internacional del Trabajo a partir de 1999 y la noción de Trabajo Digno constituye el referente normativo fundamental del ordenamiento jurídico colombiano en materia laboral. El contrato individual de trabajo se ha transformado, y en la práctica lo que se utiliza por parte de los empleadores en el sector salud es el contrato de prestación de servicios. El objetivo de la investigación es describir la vulneración de los derechos de los trabajadores del sector salud en el municipio de Soledad-Atlántico.

La estrategia metodológica utilizada para realizar esta investigación se enmarca en un enfoque descriptivo y cualitativo, básicamente en las que se inscriben en el ámbito del método hermenéutico cuya finalidad es comprender e interpretar, se utilizó como técnica el análisis documental y la entrevista. La principal conclusión a la que se llegó es la violación de los derechos laborales por parte de del Estado Colombiano al permitir este tipo de contratos teniendo en cuenta la Declaración Universal de los Derechos Humanos que plantea que toda persona tiene derecho al trabajo y a la seguridad social; el Artículo 25 de

${ }_{1}^{1}$ Abogada, especialista en Derecho Comercial y en Derecho de Familia, Magister en Psicología y consejería familiar y en Educación con énfasis en Gestión educativa, Investigadora Asociada de Colciencias (IA), Líder del Grupo Incom -Categoría A, Profesor Titular de carrera en pregrado y posgrado, Directora del Centro de Investigaciones Jurídicas y Socio-jurídicas (Universidad Libre Seccional Barranquilla). Email: sandra.villa@unilibre.edu.co

${ }^{2}$ Abogado. Doctorante en Ciencias Jurídicas en la Universidad para la Cooperación Internacional México. Magíster en Derecho Procesal de la Universidad Simón Bolívar de Barranquilla. Especialista en Derecho Laboral. Director del Grupo de investigación Andrés Bello de la Corporación Universitaria Rafael Núñez Sede Barranquilla. Email: juan.berrocal@curnvirtual.edu.co 
la Constitución Política de Colombia plantea que los trabajadores tienen derecho a un trabajo en condiciones dignas y justas.

Palabras Clave: Vulneración, derechos laborales, trabajadores, sector salud.

\begin{abstract}
The concepts of Decent Work and Decent Work are used as synonyms, however, they have different normative sources. The Decent Work approach has been developed extensively by the International Labor Organization since 1999 and the notion of Decent Work constitutes the fundamental normative reference of the Colombian legal system in labor matters. The individual work contract has been transformed, and in practice what is used by employers in the health sector is the contract for the provision of services. The objective of the research is to describe the violation of the rights of workers in the health sector in the municipality of Solitude-Atlantic.

The methodological strategy used to carry out this research is framed in a descriptive and qualitative approach, basically in those that fall within the scope of the Hermeneutical Method whose purpose is to understand and interpret, the technique used was the documentary analysis and the interview. The main conclusion reached is the violation of labor rights by the Colombian State to allow this type of contracts taking into account the Universal Declaration of Human Rights that states that everyone has the Right to Work and Social Security ; Article 25 of the Political Constitution of Colombia states that workers have the right to work in decent and fair conditions.
\end{abstract}

Keywords: Vulneration, rights, workers, health sector.

\title{
Introducción
}

T os conceptos de trabajo decente y trabajo digno, aunque en ocasiones son utilizados como sinónimos, tienen, sin embargo, fuentes normativas diferentes. El enfoque de trabajo decente ha sido desarrollado ampliamente por la Organización Internacional del Trabajo a partir de 1999 y la noción de trabajo digno constituye el referente normativo fundamental del ordenamiento jurídico Colombiano en materia laboral así lo expresa la Procuraduría General de la Nación (2010) al hacer referencia al trabajo decente, planteado por el Director General, de la OIT, Juan Somavia: 
Actualmente, la finalidad primordial de la OIT es promover oportunidades para que los hombres y las mujeres puedan conseguir un trabajo decente y productivo en condiciones de libertad, equidad, seguridad y dignidad humana. Y agregó que para cumplir con ese propósito era necesario trabajar sobre cuatro objetivos estratégicos: 1. Las oportunidades de empleo e ingresos 2. La promoción de los derechos fundamentales en el trabajo 3. La protección social 4. El diálogo social. (p.22)

A raíz de este pronunciamiento el Gobierno Nacional expidió el Decreto 2362 de 2015.

Que la Organización Internacional del Trabajo (OIT), en el marco de la $87^{\mathrm{a}}$ reunión de la Conferencia Internacional del Trabajo celebrada en el año de 1999, estableció que el Trabajo Decente abarca cuatro grandes dimensiones: el empleo (existencia de empleos suficientes), la protección social, los derechos fundamentales de los trabajadores (que incluye entre otros la libertad de sindicación y erradicación de la discriminación laboral, del trabajo forzoso y del trabajo infantil) y el diálogo social; así mismo, que el Trabajo Decente es fuente de la dignidad personal, estabilidad familiar y paz en la comunidad, actuando en beneficio de todos, en búsqueda del crecimiento económico, aumentando las oportunidades de trabajo productivo y el desarrollo de las empresas, para reducir la pobreza y obtener un desarrollo equitativo, inclusivo y sostenible.

Que en este sentido, el Trabajo Decente puede entenderse como aquella actividad productiva justamente remunerada y ejercida en condiciones de libertad, equidad, seguridad y dignidad humana, plasmado a través del respeto de los principios de igualdad de género y de no discriminación.

Si esto es así, como lo plantean la Organización Internacional del Trabajo (OIT), en el marco de la $87^{\mathrm{a}}$ reunión de la Conferencia Internacional del Trabajo celebrada en el año de 1999, y lo acordado en la Constitución 
Política de Colombia de 1991 se estaría hablando de que en Colombia debería existir una Sociedad Decente tal como lo establece Avisshai Margalit (1997) al decir que:

Una sociedad decente es aquella cuyas instituciones no humillan a las personas. Las instituciones sociales se pueden describir de dos maneras: de manera abstracta, por sus normas o leyes, o de manera concreta, por su conducta real. Análogamente se puede hablar de la humillación institucional a través de la ley, diferenciándola de los actos concretos de humillación institucional. (p. 15)

En todo caso, el control social del estamento jurídico no se dirigiría a los tipos de contratos sino a la utilización fraudulenta en general de las insti- tuciones legales en detrimento de la población trabajadora. Como sucede en Colombia, cuando se promulga la Ley 80 de 1993 que en su Artículo 32 hace referencia a los contratos estatales y entre ellos se encuentra el contrato de prestación de servicios. Estos contratos de prestación de ser- vicios, es una de las formas más burdas de vinculación laboral en Colom- bia, sobre todo en el área de los profesionales de la salud, se trata de ocultar la existencia de un verdadero contrato de trabajo ya que niega la relación laboral entre empleador y trabajador lo que impide que se produzca una serie de obligaciones legales y laborales.

Estos contratos de prestación de servicios no generan el pago de prestaciones sociales, no cuentan con el pago de vacaciones remuneradas, la seguridad social (pensión, salud y riesgos profesionales) es asumida por los contratantes, no tiene indemnización por despido sin justa causa, ni remuneración para descansos dominicales, ni tampoco beneficios adicionales y extralegales. Este tipo de contrato se sigue utilizando, a pesar de que fueron prohibidos por la Corte Constitucional en la Sentencia C-614 de 2009, al expresar esta que:

La Corte encuentra que la prohibición a la administración pública de celebrar contratos de prestación de servicios para el ejercicio de funciones de carácter permanente se ajusta a la Constitución, porque constituye una medida de protección a la relación 
Derecho humano al trabajo. Estudio de caso: los derechos de los trabajadores

laboral, ya que no sólo impide que se oculten verdaderas relaciones laborales, sino también que se desnaturalice la contratación estatal, pues el contrato de prestación de servicios es una modalidad de trabajo con el Estado de tipo excepcional, concebido como un instrumento para atender funciones ocasionales, que no hacen parte del giro ordinario de las labores encomendadas a la entidad, o siendo parte de ellas no pueden ejecutarse con empleados de planta o se requieran conocimientos especializa- dos. De igual manera, despliega los principios constitucionales de la función pública en las relaciones contractuales con el Es- tado, en tanto reitera que el ejercicio de funciones permanentes en la administración pública debe realizarse con el personal de planta, que corresponde a las personas que ingresaron a la administración mediante el concurso de méritos. (p.1)

El objetivo de esta investigación es describir la vulneración de los derechos de los trabajadores del sector salud en el Municipio de Soledad Atlántico

La metodología utilizada para el desarrollo de este estudio se enmarco en las investigaciones con enfoque descriptivo y cualitativo, básicamente en las que se inscriben en el ámbito del Método Hermenéutico descripto Martínez Migueles, (2007) que a su turno lo retoma de Dilthey uno de los principales exponentes del método hermenéutico en las ciencias humanas quien lo define en los siguientes términos:

El proceso por medio del cual conocemos la vida psíquica la ayuda de signos sensibles que son su manifestación" (1900). Es decir, que la hermenéutica tendría como misión descubrir los significados de las cosas, interpretar lo mejor posible las palabras, los escritos, los textos, los gestos y, en general, el comportamiento humano, así como cualquier acto u obra suya, pero conservando su singularidad en el contexto de que forma parte. (p. 102)

El tipo de investigación definido es de naturaleza cualitativa que de acuerdo con Bernal (2010), corresponde a un de tipo de investigación con 
enfoque cualitativo en el cual se utilizan los siguientes instrumentos otécnicas, de acuerdo con el problema objeto del estudio que se va a realizar y por ello se utilizarán técnicas como entrevistas, encuestas, análisis de texto y grupos focales, entre otras.

De acuerdo con Bernal (2010), tomado de otros autores, la población es "el conjunto de todos los elementos a los cuales se refiere la investigación”. En este caso, la población está conformada por catorce trabajadores de la salud vinculados a la E.S.E. Hospital Materno Infantil de Soledad, tales como el personal administrativo, médicos generales, auxiliares de enfermería, y enfermeras en el primer semestre del año 2017 población la cual se observa la vulneración de sus derechos laborales.

La principal conclusión a la que se llegó en la respectiva investigación es la violación de los derechos laborales por parte del Estado Colombiano al permitir este tipo de contratos teniendo en cuenta la declaración Universal de los Derechos Humanos que plantea que toda persona tiene Derecho al Trabajo y a la Seguridad Social. El Artículo 25 de la Constitución Política de Colombia plantea que los trabajadores tienen derecho a un trabajo en condiciones dignas y justas.

\section{Desarrollo}

Los resultados encontrados representan la humillación por parte del Es- tado, al expedir leyes que permiten los contratos de prestación de servicios y por la conducta asumida por la E.S.E. Hospital Materno Infantil de So- ledad a través de la contratación de sus trabajadores, haciendo caso omiso de los mandatos constitucionales.

La entrevista aplicada con los Trabajadores de la E.S.E. Hospital Materno Infantil de Soledad se realizó con la pretensión de determinar si este contrato de prestación de servicio es en realidad un verdadero contrato de trabajo, que atenta contra los Derechos Humanos del trabajador en una Sociedad Decente.

A cada aspecto de las entrevistas se le realizó un análisis particular cuyo resultado ha quedado consignado en el siguiente diálogo. Las entrevistas realizadas fueron sometidas en sus respuestas a una triangulación articulada a la percepción del investigador. 
Detalle: Entender o determinar el alcance de la aplicación de la investigación sobre el reconocimiento del contrato realidad en Colombia. En el diálogo que se presenta a continuación se muestran los resultados obtenidos con la aplicación de las entrevistas para el conocimiento del Contrato Realidad en Colombia y la posible violación de los Derechos Humanos de los Trabajadores en una Sociedad Decente. La entrevista fue aplicada en el Municipio de Soledad, Departamento del Atlántico - Colombia en la E.S.E. Hospital Materno Infantil de Soledad.

\section{Análisis de la entrevista}

1. Explique qué tipo de contrato tiene usted con la E.S.E. Hospital Materno Infantil de Soledad y desde cuando está vinculado (a)

En esta pregunta se encontró, al estudiar las repuestas ofrecidas por los trabajadores de la E.S.E. Hospital Materno Infantil de Soledad, que el 100\% de los entrevistados tiene más de dos años vinculados a la entidad con contratos de prestación de servicios.

2. ¿Dígame cuántos contratos ha realizado con la entidad y cómo ha sido la modalidad de ellos?

De acuerdo con la información suministrada por los entrevistados, un $100 \%$ asegura que los contratos son realizados cada cuatro meses, y que han realizado más de 6 contratos y todos han seguido bajo la misma modalidad del Contrato de Prestación de Servicios.

3. ¿Cuál ha sido el objeto de los contratos de prestación de servicio que ha realizado con la E.S.E. Hospital Materno Infantil de Soledad?

El 100\% de los entrevistados coinciden que su labor está acorde a la naturaleza del servicio para el que fue creado el Hospital que hace parte del giro normal de sus actividades como E.S.E. se encontraron los siguientes profesionales vinculados:

Dos (2) vacunadores, tres (3) auxiliares de archivo, un (1) psicólogo PAI, tres auxiliares de enfermería, un (1) conductor de ambulancia, dos (2) médicos generales, una (1) enfermera jefe y una (1) trabajadora social. 
4. ¿Cumple horario, o tiene asignado un cronograma de turnos con horas estipuladas de entrada y salida? ¿Y qué dificultades se le han presentado en el cumplimiento de este horario o cronograma de turnos?

El $29 \%$ de los entrevistados manifestó que se les asigna horario de trabajo, (vacunador, auxiliares de archivo, sicólogo, y a la trabajadora social), mientras que al $71 \%$ le asignan cronograma de turno (auxiliar de enfermería, medico, enfermera jefe y conductor de ambulancia). Y, por consiguiente, están obligados a cumplir el horario, o el cronograma asignado de turnos con horas estipuladas de entrada y salida. Si no se obedece se pone en riesgo el contrato

5. ¿Es continua y necesaria su presencia en la entidad prestadora de salud? ¿por qué?

El $100 \%$ de los entrevistados contestaron que su labor es indispensable para el funcionamiento de la entidad, por lo que se requiere la presencia de ellos en la jornada laboral o en los turnos fijados por la entidad. Por lo tanto, su presencia es continua y necesaria en la entidad prestadora de salud.

6. ¿Tiene jefe inmediato? ¿Recibe orden e instrucciones de este jefe inmediato? ¿Con qué frecuencia?

El 29 \% de los entrevistados respondió que sí tienen jefe inmediato, que éste les da órdenes en cualquier momento, y $71 \%$ dice que las órdenes del superior se da en cuanto la asignación de turnos y la rotación de los servicios que se prestan en el hospital como urgencias, cirugía, consulta externa, etc.

7. ¿Recibe dotación o suministro de dotación como uniformes o equipos de protección? Detállelos.

En ninguno de los casos los entrevistados manifestaron haber recibido dotación como uniformes o implementos de trabajo.

8. ¿Recibe una remuneración por honorarios periódicamente? Detállelos

$\mathrm{Si}$, manifiestan que les cancelan los honorarios pactados por la prestación del servicio más no las prestaciones sociales. 
9. ¿Se encuentra afiliado y de qué forma al régimen de Seguridad Social integral? ¿quién lo cancela en su totalidad?

La Seguridad Social integral llámese salud, pensión y riesgos laborales corren por cuenta de los trabajadores entrevistados, quienes manifiestan que para que le cancelen los honorarios deben pasar una cuenta de cobro adjuntando el pago de la seguridad social.

10. ¿Cuántas veces ha salido de vacaciones? ¿por cuánto tiempo? ¿se las han remunerado?

El 100\% de los entrevistados expresa su inconformidad, al manifestar que nunca han tenido vacaciones y mucho menos pueden pretender que éstas; les sean canceladas, pues este tipo de contrato no las contempla.

11. ¿Le cancelan los dominicales? ¿cuándo los trabaja? ¿en qué proporción?

En ningún caso, tienen la posibilidad del reconocimiento y pago de estos dominicales así se trabajen. El personal más afectado son los médicos, enfermeras y conductores de la ambulancia.

12. ¿Cada vez que termina un contrato le cancelan las prestaciones sociales?

Al responder este interrogante, el 100\% de los entrevistados manifestó que ellos no gozan de ningún tipo de prestación social como son las cesantías, intereses de cesantía, prima de servicio.

13. ¿Poseen ustedes beneficios adicionales o extralegales? Enúncielos. El

$100 \%$ de los entrevistados coinciden en manifestar que no gozan de ningún beneficio adicional o extra legal.

\section{Conclusiones}

Una de las principales conclusiones en este trabajo, se refiere a la natura- leza jurídica del contrato de prestación de servicios, ya que los contratistas vinculados a la E.S.E. Hospital Materno Infantil de Soledad, asumen di- rectamente el pago de la seguridad social, y se les niega el derecho a percibir el pago de las prestaciones sociales a las que tiene derecho por ley, tales como vacaciones, licencias por maternidad o incapacidad, pago de dominicales, cesantías, 
etc., que en realidad son desventajas para quienes son contratados bajo esta modalidad.

De acuerdo con Gómez Polo (2017), en el año "2016 había 243.427 personas que estaban contratadas en el sector público bajo la modalidad de contrato de prestación de servicios. De ahí, el $27 \%$ ha estado vinculado durante más de un año y el $17 \%$, por más de dos. Sin embargo, la cifra total de trabajadores que cotizan como independientes es cerca de 800.000".

Este contrato de prestación de servicios ha sido desnaturalizado, ya que fue concebido por el legislador colombiano en forma de temporal y se observa en la E.S.E. Hospital Materno Infantil de Soledad que ha sido utilizado para cumplir funciones permanentes que ameritan dedicación exclusiva. Así fue manifestado por todo el personal entrevistado coincidiendo que es un trabajo precario, ya que ninguno de los entrevistados goza de la seguridad social integral a la que tienen derecho; ni prestaciones sociales como primas de servicio, cesantías, intereses a las cesantías, el pago de dominicales y festivos y mucho menos a formar parte de un sindicato.

$\mathrm{Al}$ momento de presentar su cuenta de cobro con la cual le cancelan sus honorarios por la prestación del servicio, el trabajador de la E.S.E. Hospital Materno Infantil de Soledad debe anexar el pago de la seguridad social; si no lo hace, no se le cancela sus honorarios. El trabajador como contratista independiente debe aportar al Sistema de Seguridad Social de acuerdo con el artículo 135 de la ley 1753 de 2015 establece que el ingreso base de cotización al sistema de seguridad social en los trabajadores independientes no puede ser inferior al 40\% del ingreso mensual.

En su escrito publicado en el diario el espectador. Marín Correa \& Vega (2017) expresan que de acuerdo:

Con la Central Unitaria de Trabajadores (CUT), al menos el 62 $\%$ de los trabajadores que laboran en entidades públicas son contratistas. Lo que sustenta el reciente llamado de atención de la Corte Constitucional: "El uso indiscriminado de contratos de prestación de servicios constituye una violación sistemática de la Constitución”, indicó hace menos de un mes el alto tribunal. 
Derecho humano al trabajo. Estudio de caso: los derechos de los trabajadores

La Corte agregó que los poderes públicos han usado de forma abierta y amplía la figura del contrato de prestación de servicios para enmascarar relaciones laborales y evadir el pago de prestaciones sociales, desconociendo así las garantías especiales de la relación laboral que la Constitución consagra, dejando de lado, además, la excepcionalidad de este tipo de contratación.

La Corte Constitucional a través de la Sentencia C-614 de 2009, se pronunció sobre los contratos de prestación de servicios, expresando lo siguiente:

La Corte encuentra que la prohibición a la administración pública de celebrar contratos de prestación de servicios para el ejercicio de funciones de carácter permanente se ajusta a la Constitución, porque constituye una medida de protección a la relación laboral, ya que no sólo impide que se oculten verdaderas relaciones laborales, sino también que se desnaturalice la contratación estatal, pues el contrato de prestación de servicios es una modalidad de trabajo con el Estado de tipo excepcional, concebido como un instrumento para atender funciones ocasionales, que no hacen parte del giro ordinario de las labores encomendadas a la entidad, o siendo parte de ellas no pueden ejecutarse con empleados de planta o se requieran conocimientos especializados.

De acuerdo con lo manifestado por la Corte Constitucional estos contra- tos de prestación de servicios se deben celebrar con personas naturales, siempre y cuando la tarea no pueda realizarse con personal de planta o se requiera a un espe- cialista. Realizar la contratación de otra manera se considera como un fraude laboral e incumplimiento de criterios de interpretación judicial, de la Corte Constitucional.

Por otra parte, en este estudio se observa que no se tienen en cuenta los pronunciamientos de la Corte Constitucional, ya que los trabajadores de la E.S.E. Hospital Materno Infantil de Soledad tienen horario, o cumplen turnos lo que evidencia la existencia de un verdadero contrato de 
trabajo donde se presentan los tres elementos fundamentales de la relación laboral como son la presentación personal, la subordinación y el salario. Además, cumplen las mismas funciones que les son asignadas a los empleados de planta.

De acuerdo a los pronunciamientos proferidos por de la Corte Constitucional, esas son características de un contrato laboral y esos contratistas, pueden acudir a la justicia ordinaria o contenciosa administrativa a solicitar el pago de los emolumentos laborales dejados de cancelar. Según la Corte Constitucional, no pagarles sería inconstitucional e ilegal.

Por otro lado, como lo demuestra el presente estudio, la falla sigue siendo en el cumplimiento de lo sentenciado y en los controles administrativos del Estado para con sus propios organismos.

Con el propósito de cambiar esta situación, en su escrito publicado en el diario El Espectador Marín Correa, A \& Vega plantean que:

Jorge Enrique Buitrago, vicepresidente de la organización sindical Asoméritos (defensores del mérito del Estado colombiano), radicó una acción popular que fue admitida el 10 de julio por un juzgado administrativo de Bogotá. Su meta es que no se abuse de este tipo de contratos, y menos en detrimento de los ciudadanos. Para el accionante, este tipo de contratación se ha convertido en una forma de explotación laboral y política.

Armado con estos argumentos, el promotor de la acción popular pidió regular el contrato de prestación de servicios, ya que esto se presta para que los contratistas terminen siendo víctimas de explotación laboral y politica. Laboral, porque no tienen derechos a prestaciones laborales ni salariales y no tienen derecho a los mismos beneficios de los empleados de planta. Y política, porque sus contratos estánmediados por un apoyo electoral, con lo que hipotecan su pensamiento y su derecho a elegir libremente.

$\mathrm{Y}$ agrega: Es una especie de semi esclavitud lo que viven estos contratistas, pues no sólo son explotados, sino que sus ingresos son menguados por los aportes a la seguridad social que deben hacerpor su cuenta. A pesar de que este tipo de contratación tiene su prohibición constitucional 
Derecho humano al trabajo. Estudio de caso: los derechos de los trabajadores

y legal, esta figura ha sido el común denominador en las entidades públicas para labores permanentes, constituyendo en la práctica el contrato realidad.

La Declaración Universal de los Derechos Humanos, con la cual las Nacio- nes Unidas honraron a la humanidad y otorgaron a todo el mundo el dere- cho al trabajo, de acuerdo con lo expresado de los teóricos que han abor- dado esta compleja situación, entre ellos Avisshai Margalit (1997), referida a la existencia del contrato de prestación de servicio en una sociedad de- cente han considerado que el trabajo no es simplemente una herramienta para garantizar la vida digna sino un, derecho en sí y para sí mismo.

Una sociedad decente que se basa en el respeto de los Derechos Humanos esta convidada y obligada a dar trabajo a todos los individuos que la integran aun cuando los Derechos Sociales de estos estén garantizados me- diante los subsidios al desempleo, así como garantizarles unas condiciones justas y decentes en el ámbito de la sociedad.

Para que una sociedad se considere decente sus instituciones no deben humillar a las personas, deben respetar lo que se ha pactado en el interior de ella como por ejemplo, el trabajo humano en condiciones dignas y justas. Por lo tanto, el Estado no debe diseñar estrategias indignas y humillantes, que hacen difícil la vida.

Como lo expresó Avisshai Margalit (1997): "El ideal de la sociedad justa es sublime, pero difícil de poner en práctica. En cambio, el de la sociedad decente se puede materializar incluso en la vida de nuestros hijos. Es más prioritario originar una sociedad decente que una sociedad justa”.

\section{Referencias}

Bernal, C.A. (2010), Metodología de la investigación. Tercera edición. Bogotá. Pearson

Corte Constitucional. (2009). Sentencia C - 614 de 2009. Disponible en http://www.corteconstitucional.gov.co/RELATORIA/2009/C-614-09.htm. Fecha de Consulta octubre 18 de 2017 
Congreso de la Republica. (2015). Ley 1753 de 2015. Publicado en Diario Oficial No. 49.538 de 9 de junio de 2015. Disponible en: http://www.secretariasenado.gov.co/senado/basedoc/ley_1753_2015.html\#TÍTULO I. Fecha de Consulta octubre 18 de 2017

Congreso de la Republica. (1993). Ley 80 de 1993. Disponible en: http://www.alcaldiabogota.gov.co/sisjur/normas/Norma1.jsp?i=304. Fecha de Consulta octubre 18 de 2017

Procuraduría General de la República de Colombia. (2010) Trabajo digno y decente en Colombia, Seguimiento y control preventivo a las políticas públicas. Recuperado en https://www.procuraduria.gov.co/portal/media/file/Trabajo $\% 20$ digno $\% 20 y \% 20$ decente $\% 20$ en $\% 20$ Colombia_\%20Seguimiento $\% 20$ Fecha de Consulta octubre 18 de 2017

Gómez Polo G, (2017).El drama de ser trabajador por prestación de servicios. Contratados por prestación de servicios: ¿empleados de segunda?. En: El Espectador. 14 Ago. 2017 disponible en http://www.elespectador.com/noticias/politica/contratados-por-prestacion-de-servicios-empleados-de-segundaarticulo-708069. Fecha de Consulta octubre 18 de 2017

Martínez Migueles, M. (2007). Ciencia y arte en la Metodología Cualitativa. México D.F. Trillas.

Ministerio del trabajo. (2015). Decreto 2362 de 2015. Publicado en el Diario Oficial No. 49.719 de 7 de diciembre de 2015. Disponible en https://www.icbf.gov.co/cargues/avance/docs/decreto_2362_2015.htm. Fecha de consulta 16 de enero de 2018

Marín Correa, A \& Vega, J.C. (2017). Para no violar normas laborales. Buscan freno a contratos de prestación de servicios. En: El Espectador, 24 Jul. 2017. Disponible en

http://www.elespectador.com/noticias/bogota/buscan-freno-contratosde-prestacion-de-servicios-articulo-704698

Margalit, A. (1997). Trad. Carmen Castells auleda. La sociedad decente. Barcelona (España): Paidós Ibérica. 